\title{
SMAP MISSION: CHANGES IN THE RFI ENVIRONMENT
}

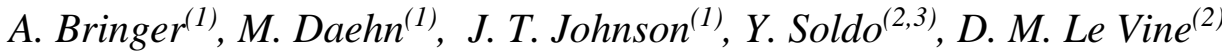 \\ P. de Matthaeis ${ }^{(2,3)}$, J. R. Piepmeier ${ }^{(2)}$, P. Mohammed ${ }^{(2,4)}$ \\ (1) ElectroScience Laboratory \\ The Ohio State University \\ Columbus, OH 43212, USA \\ (2) NASA Goddard Space Flight Center \\ Greenbelt, MD 20771 \\ (3) Universities Space Research Association, \\ Columbia, MD, USA \\ (4) Morgan State University \\ Baltimore, MD 21251
}

\begin{abstract}
The Soil Moisture Active/Passive satellite microwave radiometer has been providing measurements of L-band thermal emission from Earth for more than 2 years. SMAP retrieves surface soil moisture from its brightness temperature measurements, and continues to provide science products to the user community. Even though the SMAP radiometer operates in a protected band, its measurements are still corrupted by Radio Frequency Interference (RFI) caused by illegal in-band transmissions or out-of-band emissions. The SMAP radiometer was designed to include special hardware to enable RFI detection and filtering using multiple detection algorithms. Given the good overall performance of SMAP algorithms to detect RFI sources, an automatic tool to report source properties automatically was developed and is now operational. This paper provides a preliminary analysis of the outputs of this reporting tool with a particular focus on the evolution of the RFI environment observed by SMAP during its period of operations.
\end{abstract}

Index Terms - SMAP, Radio Frequency Interference, L-Band, RFI Detection, RFI, Mitigation

\section{INTRODUCTION}

The Soil Moisture Active/ Passive satellite (SMAP) was launched in January 2015. Since then, its L-Band radiometer has measured the thermal emission from the Earth in order to retrieve surface soil moisture and provide global soil moisture maps with a spatial resolution of $36 \mathrm{~km}$ and a temporal resolution of 3 days [1]. Even though SMAP operates in a protected band of the spectrum, other transmitters emit either within the band or in adjacent bands, causing radio frequency interference (RFI). Such interfering signals are a major concern as they corrupt the radiometer measurements and can then lead to bias in soil moisture retrievals.

Compared to previous L Band satellite radiometers, SMAP includes a digital back-end to help in detecting RFI and filtering its contributions. For each $\sim 15 \mathrm{msec}$ footprint, the SMAP radiometer provides 2 data sets in each polarization (horizontal and vertical): fullband data (32 measurements) referred to as time domain data and subband data (8 measurements for 16 frequency channels) referred as frequency domain data. In addition to the brightness temperature measured by the radiometer, the fullband and subband products also include the kurtosis and the third and fourth Stokes parameters for each measurement. RFI detection and mitigation is done in ground processing using 9 algorithms for both subband and fullband data [2].

Several studies have demonstrated the overall performance of the SMAP RFI detection and filtering processor $[3,4]$. Because of SMAP's capability to detect RFI sources, a global monitoring of the RFI environment is now possible and is an ongoing activity of the SMAP radiometer team. To support these efforts, an automatic tool has been developed as a joint effort between The Ohio State University and the NASA Goddard Space Flight Center to archive the RFI sources detected by SMAP.

This paper is focused on the analysis of the RFI environment based on the outputs of this automatic tool and how the RFI environment has changed during the two year mission. Section 2 provides first a description of the RFI reporting tool. Section 3 details the products generated by the tool. Sections 4 and 5 are focused on the analysis of the 2 year RFI source lists that have been reported to date.

\section{RFI REPORTING TOOL}

A reporting tool has been developed in order to list automatically on a weekly basis the RFI sources detected by 
the SMAP processor. The list of the sources is archived in a table that provides information on the location of the sources, as well as statistics about the RFI level and and frequencies (using the SMAP sub-bands at 1401.5 to $1425.5 \mathrm{MHz}$ ) impacted by the RFI sources. The table is the merged result of two processors. One was developed at The Ohio State University and mainly provides statistical information about the sources. The second was developed at NASA Goddard Space Flight Center in order to provide a precise location of sources as well as some information about the RFI level for both polarizations. A more detailed description of this tool can be found in [5].

Because the number of RFI sources is large, only sources with RFI levels higher than $10 \mathrm{~K}$ are reported. The most persistent sources are then selected, i.e. they must be visible in more than $25 \%$ of SMAP overpasses within a month.

The tool has been running since April 2017 and has produced weekly tables since then. The data acquired before April 2017 have been processed as well and more than 600 tables are now available for the entire mission. Analysis of these tables will help in assessing changes in the RFI environment over the two year of the mission as it will be shown in the following sections. The full results of this detailed analysis will be reported in the presentation.

\section{ANALYSIS OF THE RFI SOURCE LIST}

In addition to the tables, maps of the reported RFI sources are generated every week as well. An example map is shown in Figure 1

The color indicates the percentage in time that a source is seen, i.e. the number of observations divided by the number of SMAP overpasses over a month. The most persistent sources are appear as red in the figure. Comparing these weekly maps helps to monitor changes in time of the RFI sources. This analysis has been used to report the RFI sources to some national spectrum authorities (in China and Japan for example).

Since the tables also contain information about the flagging of each frequency channel, they allow us to track any time variations of the characteristics of the sources, in terms of frequency channels impacted by the RFI sources. This is illustrated in Figure 2, which shows the percentage of the number of pixels flagged over a month for each frequency channel as function of time for a persistent source in Mali. Note that for clarity, several channels with the same behavior as the $1406 \mathrm{MHz}$ channel were not plotted on the figures. Each data point is actually coming from a different table from July 2017 to October 2017. This figure indicates that the characteristics of this RFI source in Mali, for example a higher RFI level, changes in August when the percentage suddenly increases for all of the channels. A more detailed is needed to find out the explanation of this variation. Also, this figure was done using 19 weeks of data; the study will be completed looking at all the tables for the two years of mission operation. Further examples of this analysis will be reported in the presentation as well.
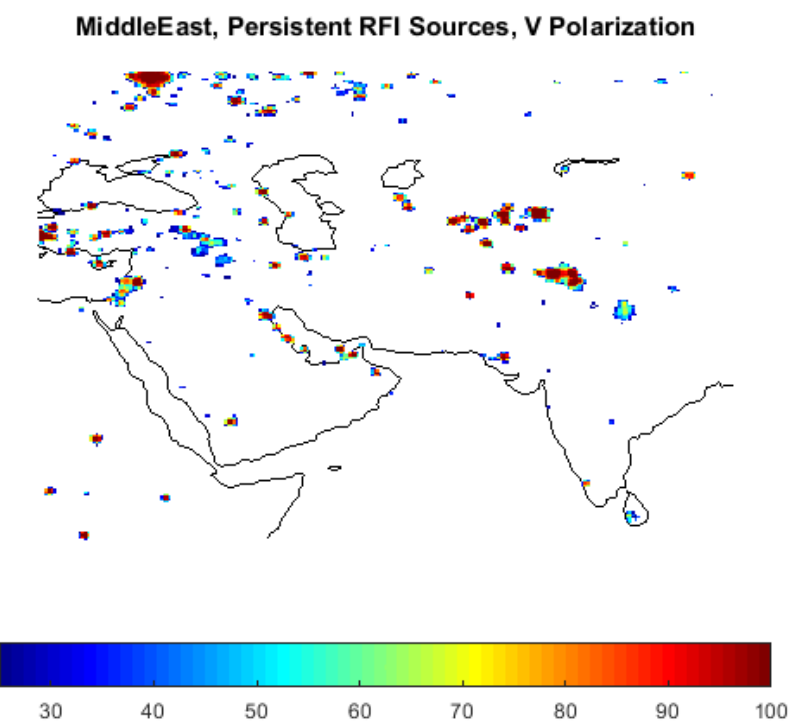

Figure 1: Persistent sources in the Middle East region for $\mathrm{V}$ polarization from July $3^{\text {rd }}$ to July $9^{\text {th }} 2017$.

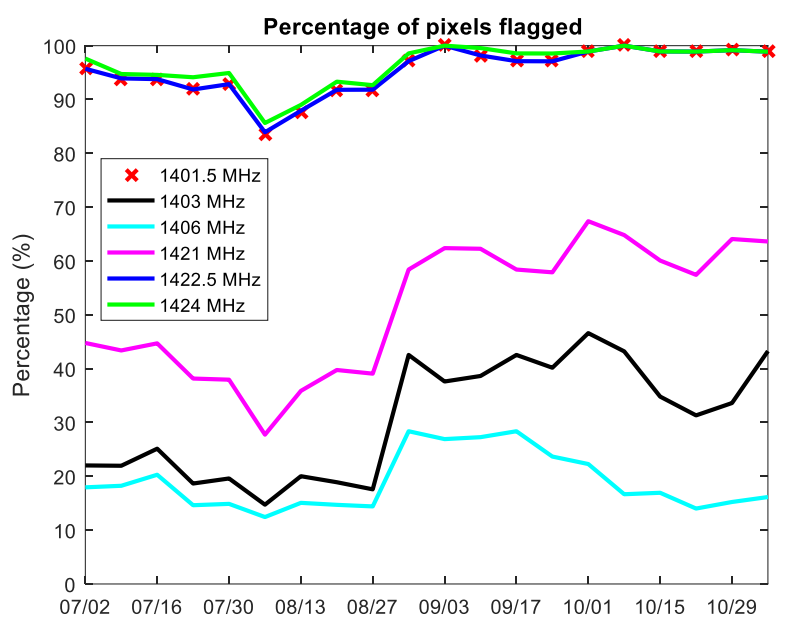

Figure 2: Evolution in time of the percentage of the number of pixels flagged channel by channel, since July $2^{\text {nd }} 2017$ for a persistent source in Mali.

\section{MONITORING CHANGES IN THE RFI ENVIRONMENT}

As the persistent source maps are generated on a weekly basis, a simple way to monitor the changes in RFI environment for a particular time period is to compute the difference between two maps of persistent sources (cf. Figure 1) generated at two different times. This operation provides an idea of the number of sources which are still present, the ones that have been disappeared, and any new sources. Week to week comparisons can be done, as well as month to month. 
An example of this difference map is presented in Figure 3, which shows the changes between November $19^{\text {th }} 2017$ and December $17^{\text {th }} 2017$ in Europe. The green color indicates no changes: the source is still present from one month to the other. Sources that disappeared during that period are shown in blue, and new sources that appear are shown in red. Sources in this example are largely stable over this period, but some changes can be observed: for example a source in Riga, Latvia disappeared during this month.

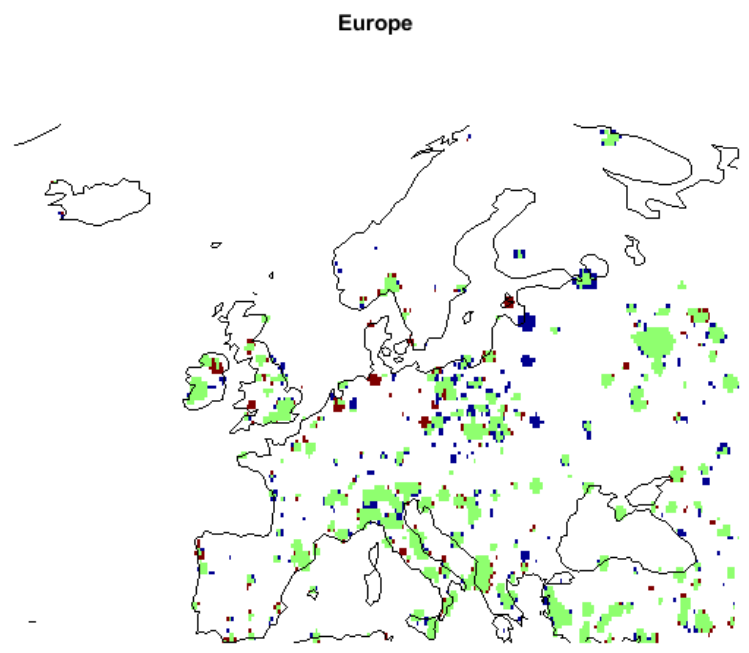

Figure 3 : Difference map of the persistent sources in Europe between November and December 2017. Green color means that the situation has not changed, the blue color that a source has disappeared and the red color that a new source has appeared.

In this paper, two other examples of significant changes observed in 2017 are presented. The first illustrates the situation in China, the second examines sources in Africa.

\subsection{China}

Numerous RFI sources have been observed in China. However, after reporting those sources to the national authorities, the situation has slightly improved in that fewer sources were observable for several months as shown in Figure $4 \mathrm{a}$. In some places (in blue on the figure), sources disappeared between July 2017 and August 2017.

The situation remained stable for approximately two months, after which new sources began to appear. Figure $4 \mathrm{~b}$ presents a comparison between the situation in August 2017 and in December 2017. A significant number of sources (red) appear during this period. A more detailed analysis will be provided in the presentation.

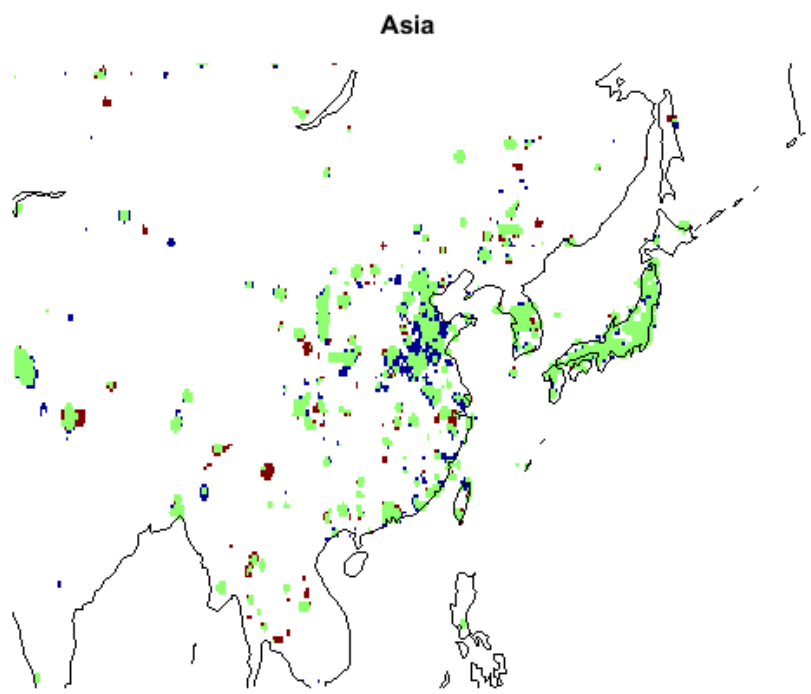

Figure 4a: Difference map of the persistent sources in Asia for the time period of July- August 2017.

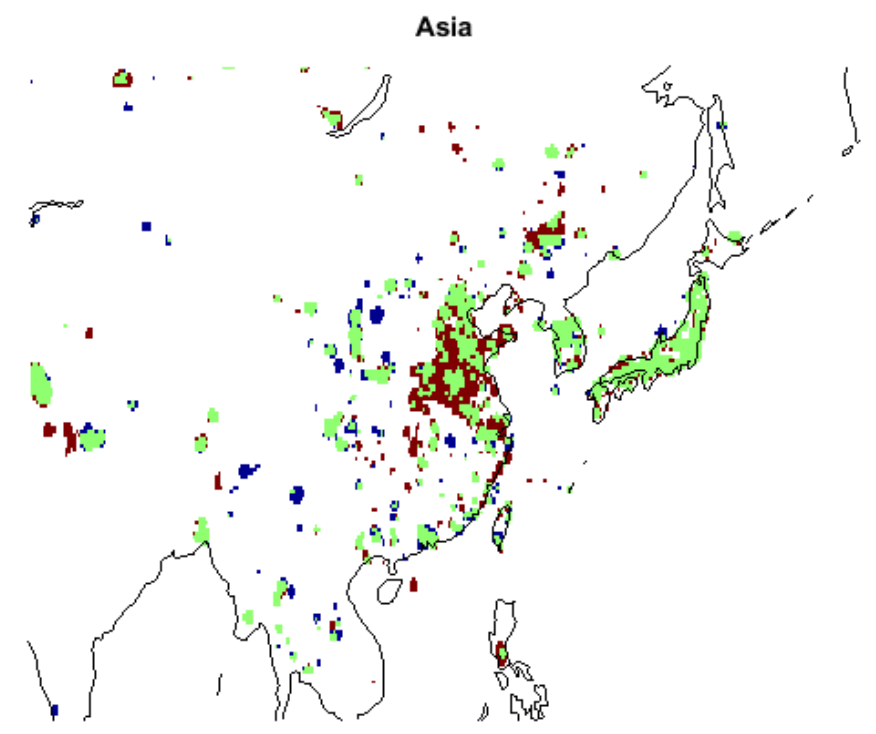

Figure 4b: Difference map of the persistent sources in Asia for the time period of August - December 2017.

\subsection{Africa}

The RFI sources in Africa are usually persistent and the situation is generally stable.

However, during Summer 2017, strong sources appeared in Northern Africa, near Cairo, Egypt as shown in Figure 5a. The situation one month later, in Figure 5b, shows that the new sources are not visible anymore as they now appear in blue in the new difference map. This example was chosen to illustrate how fast the RFI environment can change and the importance of monitoring RFI sources on a weekly basis. 


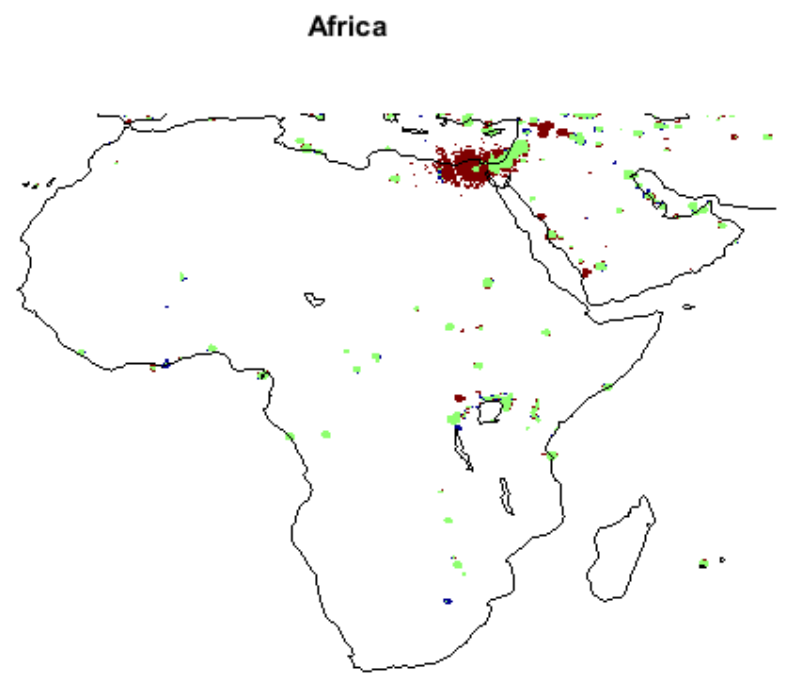

Figure 5a: Difference map of the persistent sources in Africa between August 2017 and September 2017.

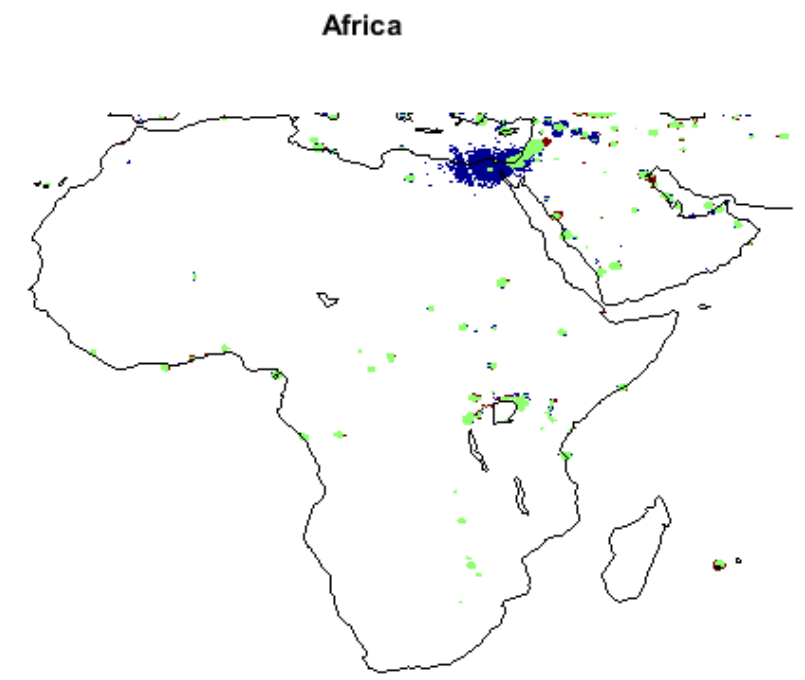

Figure 5b: Difference map of persistent sources in Africa one month later, between September 2017 and October 2017.

\section{CONCLUSION}

The good overall performance of SMAP to detect and filter RFI sources makes the monitoring of the RFI sources possible. This paper shows, through different examples, the importance of reporting those sources on a weekly basis as the RFI environment can change significantly from one week to another.

\section{REFERENCES}

[1] D. Entekhabi, et al, "The Soil Moisture Active Passive (SMAP) mission,” Proc. IEEE, vol. 98, no. 5, pp. 704-716, May 2010.
[2] J. R. Piepmeier, J. T. Johnson, P. N. Mohammed, D. Bradley, C. Ruf, M. Aksoy, R. Garcia, D. Hudson, L. Miles, and M. Wong, "Radio-Frequency Interference Mitigation for the Soil Moisture Active Passive Microwave Radiometer," IEEE Trans. Geosci. Remote Sens., vol. 42, no. 1, pp. 761-775, Jan. 2014.

[3] Mohammed, Priscilla N., et al. "SMAP L-Band Microwave Radiometer: RFI Mitigation Prelaunch Analysis and First Year OnOrbit Observations." IEEE Transactions on Geoscience and Remote Sensing 54.10 (2016): 6035-6047.

[4] A. Bringer, J.T. Johnson , P. N. Mohammed, P. de Matthaeis, J. R. Piepmeier, "Performance of SMAP Radiometer RFI Detection Algorithms and Analysis of Residual RFI Sources", IGARSS 2017.

[5] Y. Soldo, D. M. Le Vine, A. Bringer, P. N. Mohammed, P. de Matthaeis, J. R. Piepmeier, J.T. Johnson "Recent Advances to Address the RFI Issue in SMAP", submitted to IGARSS 2018. 